\title{
Bayesian Estimation of Stress Strength Reliability using Upper Record Values from Generalised Inverted Exponential Distribution
}

\author{
Ritu Kumaria*, Kalpana K. Mahajan ${ }^{\text {b }}$, Sangeeta Arorac \\ Department of Statistics \\ Panjab University, Chandigarh, India \\ E-mails: ${ }^{\mathrm{a} s t a t s . r i t u @ g m a i l . c o m, ~}{ }^{\mathrm{b}}$ mahajan_kr@pu.ac.in, ${ }^{\mathrm{c}}$ sarora131@gmail.com \\ ${ }^{*}$ Corresponding author
}

(Received December 26, 2018; Accepted April 12, 2019)

\begin{abstract}
The paper develops Bayesian estimators and HPD intervals for the stress strength reliability of generalised inverted exponential distribution using upper record values. For prior distribution, informative prior as well as non-informative prior both are considered. The Bayes estimators are obtained under both symmetric and asymmetric loss functions. A simulation study is conducted to obtain the Bayes estimates of stress strength reliability. Simulated data sets are also considered here for illustration purpose.
\end{abstract}

Keywords- Bayesian estimators, Record values, HPD intervals, Loss functions.

\section{Introduction}

The exponential distribution is the most commonly used distribution in reliability field due to its simple form and a characteristic of constant hazard rate. Let random variable $Y$ has an exponential distribution then the random variable $Z=1 / Y$ will have the inverted exponential distribution. Lin et al. (1989); Keller et al. (1982) have discussed the inverted exponential distribution. Dey (2007) considered Bayesian estimations of the parameters of inverted exponential distribution under symmetric and asymmetric loss functions. A shape parameter was introduced in the inverted exponential distribution to get the Generalised inverted exponential distribution (Abouammoh and Alshinigiti 2009). Abouammoh and Alshinigiti (2009) also pointed out that generalised inverted exponential distribution gives better fit than inverted exponential, gamma, weibull and generalised exponential distribution in many situations. Nadarajah and Kotz (2000) also discussed the generalised inverted exponential distribution. Dey and Pradhan (2014) considered generalised inverted exponential distribution under hybrid censoring. These models have applications not only in the field of reliability but are also used in the system reliability as well (Li, 2016; Deepika et al., 2017; Kumar and Ram, 2018; Li et al., 2019; Chopra and Ram, 2019).

Record values have an abundant role in daily life problems concerning data relating to numerous fields such as economics, weather and sports data. Chandler (1952) introduced the main idea of record values, inter-record times and started the statistical study of record values as a model for successive extremes in a sequence of independently and identically distributed random variables. The record values can be categorized into the lower and the upper records. An observation $X_{j}$ will be called an upper record value if its value is greater than all of previous observations (i.e., $X_{j}>$ $X_{i}$ for every $j>i$ ) and it will be called a lower record value if its value is less than all of previous observations (i.e., $X_{j}<X_{i}$ for every $j>i$ ). 
International Journal of Mathematical, Engineering and Management Sciences

Vol. 4, No. 4, 882-894, 2019

https://dx.doi.org/10.33889/IJMEMS.2019.4.4-070

A real life example using upper record values in case of generalised inverted exponential distribution is considered by Dey et al. (2016). The real data was originally proposed by Nelson (1972) consisting of 11 observations showing the times to breakdown of electrical insulating fluid subjected to 30 kilovolts. The data set is $17.05,22.66,21.02,175.88,139.07,144.12,20.46$, 43.40, 194.90, 47.30 and 7.74. The obtained upper record values from this data set are 17.05, 22.66, 175.88 and 194.90 .

The thrust of this paper is Bayesian estimation of stress-strength reliability in the generalized inverted exponential distribution based on upper record values. This problem was studied by Hussian (2013) for ordinary samples from generalised inverted exponential distribution. The stress strength reliability is the probability that the stress does not exceed the strength of a system. Let $Y$ represents the stress and $X$ represents the strength of a system then $\eta=P(Y<X)$ represents the stress strength reliability of the system. Baklizi (2008); Asgharzadeh et al. (2011); Tarvirdizade and Garehchobogh (2014); Nadar and Kizilaslan (2014); Hassan et al. (2015); Mahmoud et al. (2016) discussed stress strength reliability for different distributions using record values.

The scheme of the paper is as follows: In Section 2, an overview related to the model with distributional properties and stress strength reliability is given along with some details of upper record values. In Section 3, some concepts regarding priors, loss functions and HPD credible intervals used in the study are discussed. Bayesian estimators are derived for two different priors (non-informative and informative prior) using different loss functions viz. squared error loss function (SELF) and generalised entropy loss function (GELF). In Section 4, simulation is carried out to compute the Bayesian estimates using different configuration of sample sizes and parameters. Highest posterior density (HPD) Credible intervals along with the width of interval are also obtained in the Section 4. A simulated data set is also given in Section 5 followed by the brief discussion of the results.

\section{Model}

Let $X$ be a random variable having generalised exponential distribution (GIED), its probability density function (pdf) is

$f(x ; \alpha, \lambda)=\frac{\alpha \lambda}{x^{2}} e^{-\frac{\lambda}{x}}\left(1-e^{-\frac{\lambda}{x}}\right)^{\alpha-1} ; \quad x>0, \alpha>0, \lambda>0$,

where $\alpha$ is shape parameter and $\lambda$ is scale parameter. The corresponding cumulative distribution function (cdf) is

$F(x ; \alpha, \lambda)=1-\left(1-e^{-\frac{\lambda}{x}}\right)^{\alpha} \quad ; \quad x>0, \alpha>0, \lambda>0$.

The corresponding reliability function is

$R(x)=\left(1-e^{-\frac{\lambda}{x}}\right)^{\alpha} ; \quad x>0, \alpha>0, \lambda>0$.

Let $X$ and $Y$ are two independent random variables from two GIED with parameters $(\alpha, \lambda)$ and $(\beta, \theta)$ respectively, where $\alpha, \beta$ are shape parameters and $\lambda, \theta$ are scale parameters. Let $X$ 
International Journal of Mathematical, Engineering and Management Sciences

Vol. 4, No. 4, 882-894, 2019

https://dx.doi.org/10.33889/IJMEMS.2019.4.4-070

represents the strength and $Y$ represents the stress, then stress strength reliability $P(Y<X)$ is defined as (Krishna et al. 2017)

$$
\begin{aligned}
\eta & =P(Y<X) \\
& =1-\alpha \int_{0}^{1}(1-z)^{\alpha-1}\left(1-z^{\theta / \lambda}\right)^{\beta-1} d z .
\end{aligned}
$$

Let $x_{1}, x_{2}, \ldots, x_{n}$ be the $n$ upper record values from $\operatorname{GIED}(\alpha, \lambda)$ and $y_{1}, y_{2}, \ldots, y_{m}$ be the $m$ upper record values from $\operatorname{GIED}(\beta, \theta)$. Then the likelihood function is defined as (Arnold et al., 1998)

$L_{1}=f\left(x_{n}\right) \prod_{i=1}^{n-1} \frac{f\left(x_{i}\right)}{1-F\left(x_{i}\right)}$ and $L_{2}=g\left(y_{m}\right) \prod_{j=1}^{m-1} \frac{g\left(y_{i}\right)}{1-G\left(y_{i}\right)}$

where $f$ and $F$ are pdf and cdf of $\operatorname{GIED}(\alpha, \lambda)$ respectively and $g$ and $G$ are pdf and cdf of $\operatorname{GIED}(\beta, \theta)$ respectively. The joint likelihood function of $x_{1}, x_{2}, \ldots, x_{n}$ and $y_{1}, y_{2}, \ldots, y_{m}$ is

$$
\begin{aligned}
& L\left(x_{1}, x_{2}, \ldots, x_{n}, y_{1}, y_{2}, \ldots, y_{m}\right) \\
& =\alpha^{n} \lambda^{n} \beta^{m} \theta^{m}\left(1-e^{-\frac{\lambda}{x_{n}}}\right)^{\alpha}\left(1-e^{-\frac{\theta}{y_{n}}}\right)^{\beta} \prod_{i=1}^{n} \frac{e^{-\frac{\lambda}{x_{i}}}}{x_{i}^{2}\left(1-e^{-\frac{\lambda}{x_{i}}}\right)} \prod_{j=1}^{m} \frac{e^{-\frac{\theta}{y_{j}}}}{y_{j}^{2}\left(1-e^{-\frac{\theta}{y_{j}}}\right)} .
\end{aligned}
$$

\section{Bayesian Estimation}

In this Section, Bayes estimators for stress strength reliability are derived using upper record values in case of both informative and non-informative priors under symmetric loss function (squared error loss function) and asymmetric loss function (generalised entropy loss function). A brief introduction of loss functions, priors and HPD credible intervals is given below:

\section{Squared Error Loss Function (SELF)}

The squared error loss function (SELF) is defined as $L(\widehat{\xi}, \xi) \propto(\xi-\hat{\xi})^{2}$ where $\hat{\xi}$ is the Bayes estimator of unknown parameter $\xi$. Squared error loss function is the simplest symmetric loss function. The Bayes estimator of $\xi$ under SELF is $\hat{\xi}_{s}=E(\xi \mid \underline{x})$, where expectation is taken with respect to posterior density.

\section{General Entropy Loss Function (GELF)}

Squared error loss function (SELF) gives equal weights to under estimation and over estimation. However, in many situations under estimation is more serious than over estimations and vice versa. So, in order to overcome this difficulty another useful asymmetric loss function namely generalized entropy loss function (GELF) is used here.

Generalized entropy loss function is an asymmetric loss function and defined by Calabria and Pulcini (1996). This loss function is a generalization of the entropy loss function and defined as

$$
L(\xi, \hat{\xi}) \propto\left(\frac{\hat{\xi}}{\xi}\right)^{b}-b \ln \left(\frac{\hat{\xi}}{\xi}\right)-1
$$


International Journal of Mathematical, Engineering and Management Sciences

Vol. 4, No. 4, 882-894, 2019

https://dx.doi.org/10.33889/IJMEMS.2019.4.4-070

where $b \neq 0$. The constant $b$ determines the shape of the loss function. If $b<0$ then under estimation gets more serious than over estimation and vice-versa. Bayes estimator of $\xi$ under generalized entropy loss function is

$\hat{\xi}_{g}=\left[E\left(\xi^{-b} \mid \underline{x}\right)\right]^{(-1 / b)}$.

\section{Gamma Prior}

Gamma prior is frequently used informative prior in case of GIED (Dey and Dey, 2014; Dube et al. 2016). Assuming the parameters $\alpha, \lambda, \beta, \theta$ having independent gamma priors with respective pdfs

$p_{1}(\alpha) \propto \alpha^{c_{1}-1} e^{-d_{1} \alpha} \quad ; \alpha>0, c_{1}>0, d_{1}>0$,

$p_{2}(\lambda) \propto \lambda^{c_{2}-1} e^{-d_{2} \lambda} \quad ; \lambda>0, c_{2}>0, d_{2}>0$,

$p_{3}(\beta) \propto \beta^{c_{3}-1} e^{-d_{3} \beta} \quad ; \beta>0, c_{3}>0, d_{3}>0$,

$p_{4}(\theta) \propto \theta^{c_{4}-1} e^{-d_{4} \theta} \quad ; \theta>0, c_{4}>0, d_{4}>0$,

where $c_{i}$ 's,$d_{i}{ }^{\prime} ; i=1,2,3,4$ are hyper-parameters.

The joint prior density is

$$
\begin{array}{r}
p(\alpha, \lambda, \beta, \theta) \propto \alpha^{c_{1}-1} \lambda^{c_{2}-1} \beta^{c_{3}-1} \theta^{c_{4}-1} e^{-\left(d_{1} \alpha+d_{2} \lambda+d_{3} \beta+d_{4} \theta\right)} \\
; \alpha, \lambda, \beta, \theta>0, c_{1}, c_{2}, c_{3}, c_{4}, d_{1}, d_{2}, d_{3}, d_{4}>0 .
\end{array}
$$

\section{Remark}

If the value of all hyper parameters is zero i.e. $c_{i}=0, d_{i}=0 ; i=1,2,3,4$ then gamma priors develop non-informative priors and the joint prior density in case of non-informative prior is

$p(\alpha, \lambda, \beta, \theta) \propto \frac{1}{\alpha \lambda \beta \theta} \quad ; \alpha, \lambda, \beta, \theta>0$.

\subsection{Bayesian Estimation of $\boldsymbol{\eta}=\boldsymbol{P}(\boldsymbol{Y}<\boldsymbol{X})$ Using Gamma Prior}

The joint posterior distribution of the unknown parameters $\alpha, \lambda, \beta$ and $\theta$ given data using equation (3) is

$$
\begin{gathered}
\pi(\alpha, \lambda, \beta, \theta \mid \underline{x}, \underline{y})=\frac{L(\underline{x}, \underline{y} \mid \alpha, \lambda, \beta, \theta) p(\alpha, \lambda, \beta, \theta)}{\int_{0}^{\infty} \int_{0}^{\infty} \int_{0}^{\infty} \int_{0}^{\infty} L(\underline{x}, \underline{y} \mid \alpha, \lambda, \beta, \theta) p(\alpha, \lambda, \beta, \theta) d \alpha d \lambda d \beta d \theta}, \\
\propto \alpha^{n+c_{1}-1} \lambda^{n+c_{2}-1} \beta^{m+c_{3}-1} \theta^{m+c_{4}-1} e^{-\left(d_{1} \alpha+d_{2} \lambda+d_{3} \beta+d_{4} \theta\right)}\left(1-e^{-\frac{\lambda}{x_{n}}}\right)^{\alpha} \times \\
\quad\left(1-e^{-\frac{\theta}{y_{m}}}\right)^{\beta} \prod_{i=1}^{n} \frac{e^{-\lambda / x_{i}}}{x_{i}^{2}\left(1-e^{\left.-\lambda / x_{i}\right)}\right.} \prod_{j=1}^{m} \frac{e^{-\theta / y_{j}}}{y_{j}^{2}\left(1-e^{-\theta / y_{j}}\right)} .
\end{gathered}
$$

Since, the joint posterior distribution of $\alpha, \lambda, \beta, \theta$ in Equation (4) cannot be obtained analytically, the Markov Chain Monte Carlo (MCMC) technique is adopted to obtain the Bayes estimates and corresponding highest posterior density (HPD) credible interval of $\eta$. 
International Journal of Mathematical, Engineering and Management Sciences

Vol. 4, No. 4, 882-894, 2019

https://dx.doi.org/10.33889/IJMEMS.2019.4.4-070

The Metropolis-Hastings $(\mathrm{M}-\mathrm{H})$ algorithm can be used to generate random samples from any complex distribution of any dimension that is known up to a normalizing constant. The $\mathrm{M}-\mathrm{H}$ algorithm was established by Metropolis et al. (1953) and later extended by Hastings (1970). Gibbs sampler creates a sequence of samples from the full conditional probability distributions. The full posterior conditional distribution of parameters $\alpha, \lambda, \beta$ and $\theta$ are defined as

$$
\begin{aligned}
& \pi_{\alpha}(\alpha \mid \lambda, \beta, \theta) \propto \alpha^{n+c_{1}-1} e^{-d_{1} \alpha}\left(1-e^{-\lambda / x_{n}}\right)^{\alpha}, \\
& \pi_{\lambda}(\lambda \mid \alpha, \beta, \theta) \propto \lambda^{n+c_{2}-1} e^{-d_{2} \lambda}\left(1-e^{-\frac{\lambda}{x_{n}}}\right)^{\alpha} \prod_{i=1}^{n} \frac{e^{-\lambda / x_{i}}}{\left(1-e^{-\lambda / x_{i}}\right)} \\
& \pi_{\beta}(\beta \mid \theta, \alpha, \lambda) \propto \beta^{m+c_{2}-1} e^{-d_{3} \beta}\left(1-e^{-\theta / y_{m}}\right)^{\beta}, \\
& \pi_{\theta}(\theta \mid \beta, \alpha, \lambda) \propto \theta^{m+c_{4}-1} e^{-d_{4} \theta}\left(1-e^{-\theta / y_{m}}\right)^{\beta} \prod_{j=1}^{m} \frac{e^{-\theta / y_{j}}}{\left(1-e^{-\theta / y_{j}}\right)} .
\end{aligned}
$$

The full posterior conditional distributions of $\alpha, \beta, \lambda$ and $\theta$ in Equations (5), (6), (7) and (8) are not in known form. Hence to generate the random samples from Equations (5), (6), (7) and (8), the Metropolis-hasting algorithm is used. Since the full posterior conditional distribution of each parameter depends on some of the other parameters, the Gibbs sampler is also used here. The MCMC technique of M-H algorithm using Gibbs sampler is as follows:

(i) First take initial value $\left(\alpha_{(0)}, \lambda_{(0)}, \beta_{(0)}, \theta_{(0)}\right)$

(ii) Fix $k=1$

(iii) Generate $\alpha_{(k)}$ from $\pi_{\alpha}$ using M-H algorithm

(iv) Generate $\lambda_{(k)}$ from $\pi_{\lambda}$ using M-H algorithm

(v) Generate $\beta_{(k)}$ from $\pi_{\beta}$ using M-H algorithm

(vi) Generate $\theta_{(k)}$ from $\pi_{\theta}$ using $\mathrm{M}-\mathrm{H}$ algorithm

(vii) Obtain $\eta_{k}$

(viii) Set $k=k+1$

(ix) Repeat step (i) to (viii) $N$ times.

The Bayes estimator of $\eta$ under squared error loss function is $\eta_{s e l f}=\frac{1}{N-N_{0}} \sum_{r=N_{0}+1}^{N} \eta_{(r)}$. The Bayes estimator of $\eta$ under generalised entropy loss function is $\eta_{g e l f}=\left[\frac{1}{N-N_{0}} \sum_{r=N_{0}+1}^{N} \eta_{(r)}^{-b}\right]^{-1 / b}$. Note that $N_{0}$ is the burn in period.

\section{HPD Credible Intervals}

Chen and Shao (1999) introduced the algorithm to find the highest posterior density (HPD) credible intervals. $100(1-\gamma) \%$ HPD credible interval is that $100(1-\gamma) \%$ credible interval which is having smallest width among all possible $100(1-\gamma) \%$ credible intervals.

Once the posterior sample is generated for $\eta_{i}\left(i=1,2, \ldots,\left(N-N_{0}\right)\right)$, then $\eta_{(1)} \leq \eta_{(2)} \leq \cdots \leq$

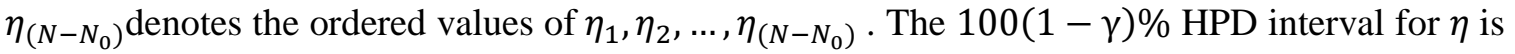
defined by $\left.\left(\eta_{(j)}, \eta_{\left(j+\left[(1-\gamma)\left(N-N_{0}\right)\right]\right)}\right)\right)$, where $j$ is chosen such that

$\eta_{\left(j+\left[(1-\gamma)\left(N-N_{0}\right)\right]\right)}-\eta_{(j)}=\min _{1 \leq j \leq M}\left(\eta_{\left(j+\left[(1-\gamma)\left(N-N_{0}\right)\right]\right)}-\eta_{(j)}\right), \quad j=1,2, \ldots,\left(N-N_{0}\right)$, where $[x]$ is the greatest integer of $x$. 
International Journal of Mathematical, Engineering and Management Sciences

Vol. 4, No. 4, 882-894, 2019

https://dx.doi.org/10.33889/IJMEMS.2019.4.4-070

\section{Simulation Study}

In this section, Monte Carlo simulation study is made for Bayes estimates for stress strength reliability of generalised inverted exponential distribution using upper record values as the estimators cannot be obtained theoretically. The Bayesian estimation is done for both informative (gamma prior) as well as non-informative prior under squared error loss function (SELF) and generalised entropy loss function (GELF) based on 3000 replications. For generalised entropy loss function the value of $b$ is taken 0.5 (for over estimation) and -0.5 (for under estimation). The values of hyper parameters $\left(c_{i}^{\prime} s, d_{i}^{\prime} s ; i=1,2,3,4\right)$ are chosen such that the true value of parameters is equal to the prior mean. Three cases of stress strength reliability are considered as small, medium and large value by taking different configurations of the true values underlying presents (i) $\eta=0.2610697(\alpha=4, \lambda=5, \beta=1, \theta=4)$ (ii) $\eta=0.5(\alpha=1, \lambda=2, \beta=1, \theta=$ 2) (iii) $\eta=0.7428572(\alpha=1, \lambda=2, \beta=2, \theta=1.5)$ respectively.

For simulation, different sample sizes are 5,10,15 and all combinations of these sample sizes are considered for $X$ and $Y$. For simulation study MCMC technique of M-H algorithm using Gibbs sampler is used. For which a chain of 20,000 observations is generated with 5000 burn-in period i.e. first 5000 observations are discarded as burn-in period from 20,000 observations. This burnin period decided by cumulative mean plots and for a simulated data set trace plots, cumulative mean plots and density plots are shown in Section 5.

Tables 1 and 2 represents the Bayes estimates, Expected loss function (in brackets), 95\% HPD credible intervals (in brackets) and their length for stress strength reliability of Generalised inverted exponential distribution using informative and non-informative prior. The value of expected loss function and the length of HPD credible intervals decrease as the sample sizes increase (Tables 1 and 2). As sample size $n, m$ increases, the loss function decreases as is seen from the Tables 1 and 2. The length of 95\% HPD credible intervals decreases as sample size increases for the Bayes estimates of stress strength reliability of generalised inverted exponential distribution. As seen from the Tables 1 and 2, the length of credible intervals for gamma prior is less than for non-informative prior. 
International Journal of Mathematical, Engineering and Management Sciences

Vol. 4, No. 4, 882-894, 2019

https://dx.doi.org/10.33889/IJMEMS.2019.4.4-070

Table 1. Bayes Estimates, Expected loss functions (in brackets), HPD credible intervals (in brackets) with length for $\eta=P(Y<X)$ using Gamma prior under squared error loss function (SELF) and Generalised entropy loss function (GELF)

\begin{tabular}{|c|c|c|c|c|c|}
\hline$P(Y<X)$ & $n, m$ & Self & $\begin{array}{c}\text { Gelf } \\
b=0.5\end{array}$ & $\begin{array}{c}\text { Gelf } \\
b=-0.5\end{array}$ & $\begin{array}{c}\text { HPD } \\
\text { credible interval }\end{array}$ \\
\hline \multirow{9}{*}{0.2610697} & 5,5 & $\begin{array}{c}0.273444 \\
(0.002636)\end{array}$ & $\begin{array}{c}0.263506 \\
(0.005437)\end{array}$ & $\begin{array}{c}0.270215 \\
(0.005083)\end{array}$ & $\begin{array}{c}(0.196111,0.410094) \\
0.213983\end{array}$ \\
\hline & 5,10 & $\begin{array}{c}0.273670 \\
(0.002540)\end{array}$ & $\begin{array}{c}0.263769 \\
(0.005275)\end{array}$ & $\begin{array}{c}0.270451 \\
(0.004959)\end{array}$ & $\begin{array}{c}(0.211205,0.425026) \\
0.213821\end{array}$ \\
\hline & 5,15 & $\begin{array}{c}0.273884 \\
(0.002485)\end{array}$ & $\begin{array}{c}0.263955 \\
(0.005147)\end{array}$ & $\begin{array}{c}0.270657 \\
(0.004825)\end{array}$ & $\begin{array}{c}(0.225881,0.439613) \\
0.213732\end{array}$ \\
\hline & 10,5 & $\begin{array}{c}0.272456 \\
(0.002626)\end{array}$ & $\begin{array}{c}0.262591 \\
(0.005562)\end{array}$ & $\begin{array}{c}0.269249 \\
(0.005247)\end{array}$ & $\begin{array}{c}(0.147477,0.359719) \\
0.212242\end{array}$ \\
\hline & 10,10 & $\begin{array}{c}0.272654 \\
(0.002621)\end{array}$ & $\begin{array}{c}0.262803 \\
(0.005543)\end{array}$ & $\begin{array}{c}0.269452 \\
(0.005226)\end{array}$ & $\begin{array}{c}(0.165999,0.374616) \\
0.208617\end{array}$ \\
\hline & 10,15 & $\begin{array}{c}0.272901 \\
(0.002513)\end{array}$ & $\begin{array}{c}0.263040 \\
(0.005292)\end{array}$ & $\begin{array}{c}0.269695 \\
(0.004976)\end{array}$ & $\begin{array}{c}(0.221307,0.426759) \\
0.205452\end{array}$ \\
\hline & 15,5 & $\begin{array}{c}0.274074 \\
(0.002546)\end{array}$ & $\begin{array}{c}0.264252 \\
(0.005216)\end{array}$ & $\begin{array}{c}0.270569 \\
(0.004887)\end{array}$ & $\begin{array}{c}(0.205698,0.416669) \\
0.210971\end{array}$ \\
\hline & 15,10 & $\begin{array}{c}0.274202 \\
(0.002528) \\
\end{array}$ & $\begin{array}{c}0.263927 \\
(0.005196) \\
\end{array}$ & $\begin{array}{c}0.270882 \\
(0.004881) \\
\end{array}$ & $\begin{array}{c}(0.240128,0.448922) \\
0.208794 \\
\end{array}$ \\
\hline & 15,15 & $\begin{array}{c}0.273768 \\
(0.002500) \\
\end{array}$ & $\begin{array}{c}0.264418 \\
(0.005172) \\
\end{array}$ & $\begin{array}{c}0.271022 \\
(0.004848)\end{array}$ & $\begin{array}{c}(0.211550,0.416144) \\
0.204594\end{array}$ \\
\hline \multirow{9}{*}{0.5} & 5,5 & $\begin{array}{c}0.495512 \\
(0.004528) \\
\end{array}$ & $\begin{array}{c}0.486167 \\
(0.002760) \\
\end{array}$ & $\begin{array}{c}0.492473 \\
(0.002638) \\
\end{array}$ & $\begin{array}{c}(0.348259,0.639513) \\
0.291254 \\
\end{array}$ \\
\hline & 5,10 & $\begin{array}{c}0.497939 \\
(0.004481) \\
\end{array}$ & $\begin{array}{c}0.485547 \\
(0.002693)\end{array}$ & $\begin{array}{c}0.491900 \\
(0.002551)\end{array}$ & $\begin{array}{c}(0.349508,0.639520) \\
0.290012\end{array}$ \\
\hline & 5,15 & $\begin{array}{c}0.494961 \\
(0.004459)\end{array}$ & $\begin{array}{c}0.488664 \\
(0.002667)\end{array}$ & $\begin{array}{c}0.494924 \\
(0.002544)\end{array}$ & $\begin{array}{c}(0.351984,0.641812) \\
0.289828\end{array}$ \\
\hline & 10,5 & $\begin{array}{c}0.497073 \\
(0.004481) \\
\end{array}$ & $\begin{array}{c}0.483188 \\
(0.002753) \\
\end{array}$ & $\begin{array}{c}0.489477 \\
(0.002619) \\
\end{array}$ & $\begin{array}{c}(0.348236,0.637923) \\
0.289687 \\
\end{array}$ \\
\hline & 10,10 & $\begin{array}{c}0.492506 \\
(0.004417)\end{array}$ & $\begin{array}{c}0.487839 \\
(0.002677)\end{array}$ & $\begin{array}{c}0.494069 \\
(0.002551)\end{array}$ & $\begin{array}{c}(0.351661,0.640565) \\
0.288904\end{array}$ \\
\hline & 10,15 & $\begin{array}{c}0.493904 \\
(0.004265) \\
\end{array}$ & $\begin{array}{c}0.484572 \\
(0.002621) \\
\end{array}$ & $\begin{array}{r}0.490869 \\
(0.002489) \\
\end{array}$ & $\begin{array}{c}(0.347330,0.636145) \\
0.288815 \\
\end{array}$ \\
\hline & 15,5 & $\begin{array}{c}0.494918 \\
(0.004327) \\
\end{array}$ & $\begin{array}{c}0.482723 \\
(0.002695) \\
\end{array}$ & $\begin{array}{c}0.488973 \\
(0.002570) \\
\end{array}$ & $\begin{array}{c}(0.349362,0.638394) \\
0.289032 \\
\end{array}$ \\
\hline & 15,10 & $\begin{array}{c}0.491984 \\
(0.004276) \\
\end{array}$ & $\begin{array}{c}0.485690 \\
(0.002633) \\
\end{array}$ & $\begin{array}{c}0.491918 \\
(0.002504) \\
\end{array}$ & $\begin{array}{c}(0.349640,0.638077) \\
0.288437 \\
\end{array}$ \\
\hline & 15,15 & $\begin{array}{c}0.494790 \\
(0.004212)\end{array}$ & $\begin{array}{c}0.485524 \\
(0.002573)\end{array}$ & $\begin{array}{c}0.491777 \\
(0.002448)\end{array}$ & $\begin{array}{c}(0.347171,0.635361) \\
0.28819\end{array}$ \\
\hline \multirow{9}{*}{0.7428572} & 5,5 & $\begin{array}{c}0.765168 \\
(0.001588)\end{array}$ & $\begin{array}{c}0.763376 \\
(0.000357)\end{array}$ & $\begin{array}{c}0.764575 \\
(0.000354)\end{array}$ & $\begin{array}{c}(0.683109,0.843801) \\
0.160692\end{array}$ \\
\hline & 5,10 & $\begin{array}{c}0.765265 \\
(0.001557)\end{array}$ & $\begin{array}{c}0.763486 \\
(0.000351)\end{array}$ & $\begin{array}{c}0.764677 \\
(0.000348)\end{array}$ & $\begin{array}{c}(0.683309,0.843778) \\
0.160469\end{array}$ \\
\hline & 5,15 & $\begin{array}{c}0.765211 \\
(0.001517)\end{array}$ & $\begin{array}{c}0.763425 \\
(0.000341)\end{array}$ & $\begin{array}{c}0.764620 \\
(0.000338)\end{array}$ & $\begin{array}{c}(0.683378,0.843605) \\
0.160227\end{array}$ \\
\hline & 10,5 & $\begin{array}{c}0.765190 \\
(0.001607) \\
\end{array}$ & $\begin{array}{c}0.763417 \\
(0.000362) \\
\end{array}$ & $\begin{array}{c}0.764604 \\
(0.000358) \\
\end{array}$ & $\begin{array}{c}(0.683859,0.844123) \\
0.160264\end{array}$ \\
\hline & 10,10 & $\begin{array}{c}0.765692 \\
(0.001575) \\
\end{array}$ & $\begin{array}{c}0.763911 \\
(0.000354) \\
\end{array}$ & $\begin{array}{c}0.765103 \\
(0.000351) \\
\end{array}$ & $\begin{array}{c}(0.683600,0.843524) \\
0.159924 \\
\end{array}$ \\
\hline & 10,15 & $\begin{array}{c}0.766062 \\
(0.001514) \\
\end{array}$ & $\begin{array}{c}0.764306 \\
(0.000339) \\
\end{array}$ & $\begin{array}{c}0.765481 \\
(0.000336) \\
\end{array}$ & $\begin{array}{c}(0.684805,0.844128) \\
0.159323 \\
\end{array}$ \\
\hline & 15,5 & $\begin{array}{c}0.765232 \\
(0.001506)\end{array}$ & $\begin{array}{c}0.763471 \\
(0.000339)\end{array}$ & $\begin{array}{c}0.764649 \\
(0.000336)\end{array}$ & $\begin{array}{c}(0.683431,0.843602) \\
0.160171\end{array}$ \\
\hline & 15,10 & $\begin{array}{c}0.765199 \\
(0.001483)\end{array}$ & $\begin{array}{c}0.763422 \\
(0.000334)\end{array}$ & $\begin{array}{c}0.764611 \\
(0.000330)\end{array}$ & $\begin{array}{c}(0.683812,0.843275) \\
0.159463\end{array}$ \\
\hline & 15,15 & $\begin{array}{c}0.766407 \\
(0.001458)\end{array}$ & $\begin{array}{c}0.764653 \\
(0.000324)\end{array}$ & $\begin{array}{c}0.765826 \\
(0.000321)\end{array}$ & $\begin{array}{c}(0.685010,0.844318) \\
0.159308\end{array}$ \\
\hline
\end{tabular}


International Journal of Mathematical, Engineering and Management Sciences

Vol. 4, No. 4, 882-894, 2019

https://dx.doi.org/10.33889/IJMEMS.2019.4.4-070

Table 2. Bayes estimates, expected loss functions (in brackets), HPD credible intervals (in brackets) with length for $\eta=P(Y<X)$ using non-informative prior under squared error loss function (SELF) and Generalised entropy loss function (GELF)

\begin{tabular}{|c|c|c|c|c|c|}
\hline$P(Y<X)$ & $n, m$ & Self & $\begin{array}{c}\text { Gelf } \\
b=0.5\end{array}$ & $\begin{array}{c}\text { Gelf } \\
b=-0.5\end{array}$ & $\begin{array}{c}\text { HPD } \\
\text { credible interval }\end{array}$ \\
\hline \multirow{9}{*}{0.2610697} & 5,5 & $\begin{array}{c}0.330588 \\
(0.027847)\end{array}$ & $\begin{array}{c}0.292779 \\
(0.040880)\end{array}$ & $\begin{array}{c}0.299357 \\
(0.047606)\end{array}$ & $\begin{array}{c}(0.163842,0.386980) \\
0.223138\end{array}$ \\
\hline & 5,10 & $\begin{array}{c}0.316604 \\
(0.024561)\end{array}$ & $\begin{array}{c}0.307051 \\
(0.035493)\end{array}$ & $\begin{array}{c}0.313513 \\
(0.035490)\end{array}$ & $\begin{array}{c}(0.163327,0.386244) \\
0.222917\end{array}$ \\
\hline & 5,15 & $\begin{array}{c}0.302498 \\
(0.020758)\end{array}$ & $\begin{array}{c}0.321416 \\
(0.031401)\end{array}$ & $\begin{array}{c}0.327608 \\
(0.028417)\end{array}$ & $\begin{array}{c}(0.163577,0.386408) \\
0.222831\end{array}$ \\
\hline & 10,5 & $\begin{array}{c}0.321324 \\
(0.037643)\end{array}$ & $\begin{array}{c}0.257222 \\
(0.045580)\end{array}$ & $\begin{array}{c}0.264560 \\
(0.052686)\end{array}$ & $\begin{array}{c}(0.163283,0.385450) \\
0.222167\end{array}$ \\
\hline & 10,10 & $\begin{array}{c}0.268040 \\
(0.020697)\end{array}$ & $\begin{array}{c}0.312146 \\
(0.044136)\end{array}$ & $\begin{array}{c}0.318333 \\
(0.043904)\end{array}$ & $\begin{array}{c}(0.162902,0.384972) \\
0.22207\end{array}$ \\
\hline & 10,15 & $\begin{array}{c}0.248681 \\
(0.009501)\end{array}$ & $\begin{array}{c}0.238041 \\
(0.019426)\end{array}$ & $\begin{array}{c}0.245243 \\
(0.018168)\end{array}$ & $\begin{array}{c}(0.163185,0.385076) \\
0.221891\end{array}$ \\
\hline & 15,5 & $\begin{array}{c}0.341742 \\
(0.043895)\end{array}$ & $\begin{array}{c}0.332159 \\
(0.044924)\end{array}$ & $\begin{array}{c}0.308685 \\
(0.044415)\end{array}$ & $\begin{array}{c}(0.164112,0.386470) \\
0.222358\end{array}$ \\
\hline & 15,10 & $\begin{array}{c}0.311732 \\
(0.037293) \\
\end{array}$ & $\begin{array}{c}0.302302 \\
(0.044886) \\
\end{array}$ & $\begin{array}{c}0.338659 \\
(0.039764) \\
\end{array}$ & $\begin{array}{c}(0.164594,0.386692) \\
0.222098 \\
\end{array}$ \\
\hline & 15,15 & $\begin{array}{c}0.308061 \\
(0.021923)\end{array}$ & $\begin{array}{c}0.298821 \\
(0.029377)\end{array}$ & $\begin{array}{c}0.305079 \\
(0.028702)\end{array}$ & $\begin{array}{c}(0.164699,0.386334) \\
0.221635\end{array}$ \\
\hline \multirow{9}{*}{0.5} & 5,5 & $\begin{array}{c}0.495526 \\
(0.005234) \\
\end{array}$ & $\begin{array}{c}0.485572 \\
(0.003242) \\
\end{array}$ & $\begin{array}{c}0.492299 \\
(0.003096) \\
\end{array}$ & $\begin{array}{c}(0.345320,0.643287) \\
0.297967 \\
\end{array}$ \\
\hline & 5,10 & $\begin{array}{c}0.495996 \\
(0.005215)\end{array}$ & $\begin{array}{c}0.486049 \\
(0.003224) \\
\end{array}$ & $\begin{array}{c}0.491976 \\
(0.003088) \\
\end{array}$ & $\begin{array}{c}(0.345742,0.643704) \\
0.297962\end{array}$ \\
\hline & 5,15 & $\begin{array}{c}0.495208 \\
(0.005188)\end{array}$ & $\begin{array}{c}0.485235 \\
(0.003233)\end{array}$ & $\begin{array}{c}0.492771 \\
(0.003080)\end{array}$ & $\begin{array}{c}(0.345755,0.643403) \\
0.297648\end{array}$ \\
\hline & 10,5 & $\begin{array}{c}0.494823 \\
(0.004988)\end{array}$ & $\begin{array}{c}0.483252 \\
(0.003110)\end{array}$ & $\begin{array}{c}0.491620 \\
(0.002959)\end{array}$ & $\begin{array}{c}(0.343675,0.641000) \\
0.297325\end{array}$ \\
\hline & 10,10 & $\begin{array}{c}0.493200 \\
(0.004924)\end{array}$ & $\begin{array}{c}0.484943 \\
(0.003107)\end{array}$ & $\begin{array}{c}0.489974 \\
(0.002958)\end{array}$ & $\begin{array}{c}(0.345355,0.641591) \\
0.296236\end{array}$ \\
\hline & 10,15 & $\begin{array}{c}0.495536 \\
(0.004871) \\
\end{array}$ & $\begin{array}{c}0.485719 \\
(0.002983) \\
\end{array}$ & $\begin{array}{c}0.492352 \\
(0.002831) \\
\end{array}$ & $\begin{array}{c}(0.346369,0.642515) \\
0.296146 \\
\end{array}$ \\
\hline & 15,5 & $\begin{array}{c}0.493530 \\
(0.005089)\end{array}$ & $\begin{array}{c}0.483702 \\
(0.003195) \\
\end{array}$ & $\begin{array}{c}0.490344 \\
(0.003045)\end{array}$ & $\begin{array}{c}(0.344291,0.640139) \\
0.295848\end{array}$ \\
\hline & 15,10 & $\begin{array}{c}0.493086 \\
(0.004956)\end{array}$ & $\begin{array}{c}0.483207 \\
(0.003133)\end{array}$ & $\begin{array}{c}0.489883 \\
(0.002985)\end{array}$ & $\begin{array}{c}(0.346976,0.642765) \\
0.295789\end{array}$ \\
\hline & 15,15 & $\begin{array}{c}0.495895 \\
(0.004793) \\
\end{array}$ & $\begin{array}{c}0.486103 \\
(0.002955) \\
\end{array}$ & $\begin{array}{c}0.492720 \\
(0.002813) \\
\end{array}$ & $\begin{array}{c}(0.344775,0.640118) \\
0.295343 \\
\end{array}$ \\
\hline \multirow{9}{*}{0.7428572} & 5,5 & $\begin{array}{c}0.765669 \\
(0.001700)\end{array}$ & $\begin{array}{c}0.763840 \\
(0.000383)\end{array}$ & $\begin{array}{c}0.765064 \\
(0.000379)\end{array}$ & $\begin{array}{c}(0.683228,0.845960) \\
0.162732\end{array}$ \\
\hline & 5,10 & $\begin{array}{c}0.766207 \\
(0.001690)\end{array}$ & $\begin{array}{c}0.764368 \\
(0.000378)\end{array}$ & $\begin{array}{c}0.765599 \\
(0.000374)\end{array}$ & $\begin{array}{c}(0.682781,0.845004) \\
0.162223\end{array}$ \\
\hline & 5,15 & $\begin{array}{c}0.767360 \\
(0.001648)\end{array}$ & $\begin{array}{c}0.765543 \\
(0.000368)\end{array}$ & $\begin{array}{c}0.766759 \\
(0.000365)\end{array}$ & $\begin{array}{c}(0.684803,0.846526) \\
0.161723\end{array}$ \\
\hline & 10,5 & $\begin{array}{c}0.764474 \\
(0.001706)\end{array}$ & $\begin{array}{c}0.762627 \\
(0.000386)\end{array}$ & $\begin{array}{c}0.763863 \\
(0.000382) \\
\end{array}$ & $\begin{array}{c}(0.681331,0.844183) \\
0.162852 \\
\end{array}$ \\
\hline & 10,10 & $\begin{array}{c}0.764813 \\
(0.001703) \\
\end{array}$ & $\begin{array}{c}0.762986 \\
(0.000385) \\
\end{array}$ & $\begin{array}{c}0.764209 \\
(0.000382) \\
\end{array}$ & $\begin{array}{c}(0.682096,0.844474) \\
0.162378 \\
\end{array}$ \\
\hline & 10,15 & $\begin{array}{c}0.765040 \\
(0.001680)\end{array}$ & $\begin{array}{c}0.763208 \\
(0.000380)\end{array}$ & $\begin{array}{c}0.764434 \\
(0.000377)\end{array}$ & $\begin{array}{c}(0.682083,0.844150) \\
0.162067\end{array}$ \\
\hline & 15,5 & $\begin{array}{c}0.765746 \\
(0.001634)\end{array}$ & $\begin{array}{c}0.763941 \\
(0.000366)\end{array}$ & $\begin{array}{c}0.765149 \\
(0.000362)\end{array}$ & $\begin{array}{c}(0.683577,0.845638) \\
0.162061\end{array}$ \\
\hline & 15,10 & $\begin{array}{c}0.766083 \\
(0.001580)\end{array}$ & $\begin{array}{c}0.764269 \\
(0.000354) \\
\end{array}$ & $\begin{array}{c}0.765483 \\
(0.000351)\end{array}$ & $\begin{array}{c}(0.683610,0.845371) \\
0.161761\end{array}$ \\
\hline & 15,15 & $\begin{array}{c}0.766305 \\
(0.001562)\end{array}$ & $\begin{array}{c}0.764485 \\
(0.000350)\end{array}$ & $\begin{array}{c}0.765703 \\
(0.000347)\end{array}$ & $\begin{array}{c}0.683488,0.844843) \\
0.161355\end{array}$ \\
\hline
\end{tabular}


International Journal of Mathematical, Engineering and Management Sciences

Vol. 4, No. 4, 882-894, 2019

https://dx.doi.org/10.33889/IJMEMS.2019.4.4-070

\section{Simulated Data Sets}

Two simulated data sets $x$ and $y$ are considered from $\operatorname{GIED}(\alpha, \lambda)$ and $\operatorname{GIED}(\beta, \theta)$ taking $\alpha=$ $3, \lambda=3, \beta=2, \theta=2$ and $\underline{r}, \underline{s}$ are upper record values from $x, y$ respectively. In M-H algorithm we have generated chain of 20,000 observations taking burn-in period as 5000 i.e. discards the first 5000 observations. The trace plots (Figure 1) show the randomness of observations $(\alpha, \lambda, \beta, \theta)$ and the convergence of chain presented by the cumulative mean plots (Figure 2). The density plots for $(\alpha, \lambda, \beta, \theta)$ are presented in Figure 3 .

$x=1.3333,1.6369,1.2695,1.7448,3.7180,3.1199,1.9989,10.3106,1.0055,0.8094$

$\underline{r}=1.3333,1.6369,1.7448,3.7180,10.3106$

$y=1.1843,2.1003,1.0131,0.9735,3.3223,0.3973,2.3858,9.3776,2.2003,1.8625$

$\underline{s}=1.1843,2.1003,3.3223,9.3776$.

trace plot for alpha

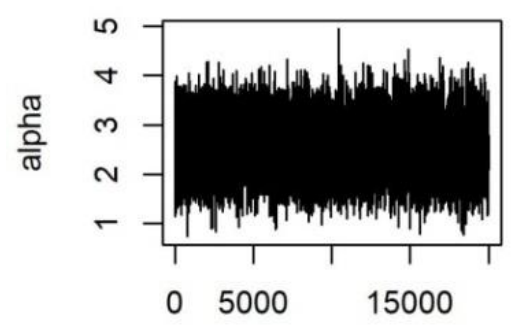

iterations

trace plot for beta

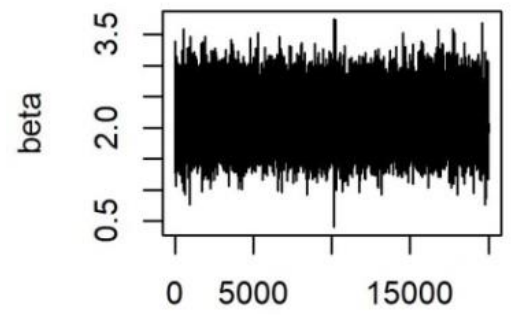

iterations trace plot for lambda

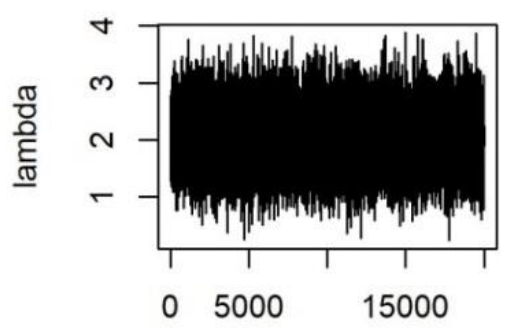

iterations

trace plot for theta

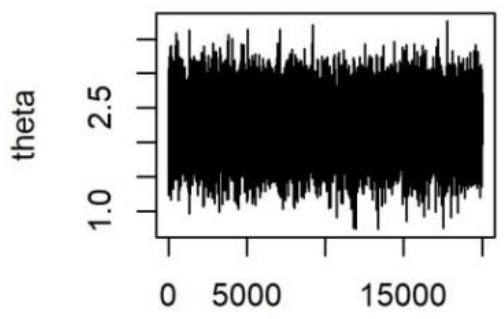

iterations

Figure 1. Trace plots for $\alpha, \lambda, \beta, \theta$. 
International Journal of Mathematical, Engineering and Management Sciences

Vol. 4, No. 4, 882-894, 2019

https://dx.doi.org/10.33889/IJMEMS.2019.4.4-070

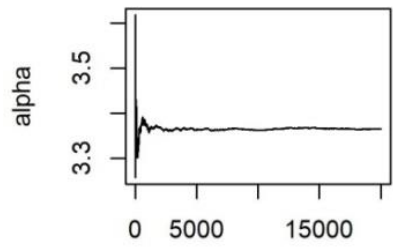

iterations

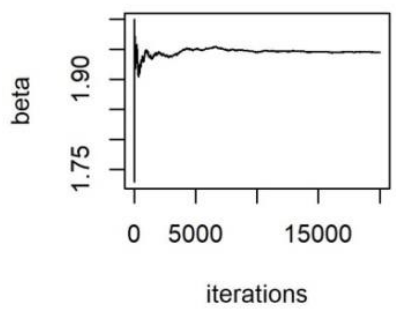

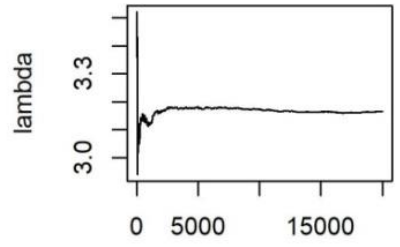

iterations

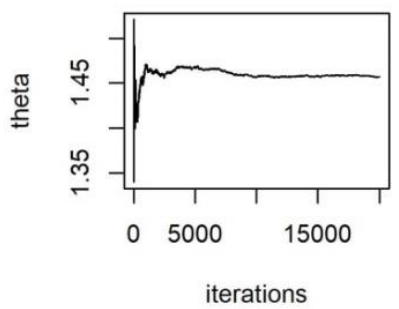

Figure 2. Cumulative mean plots for $\alpha, \lambda, \beta, \theta$.

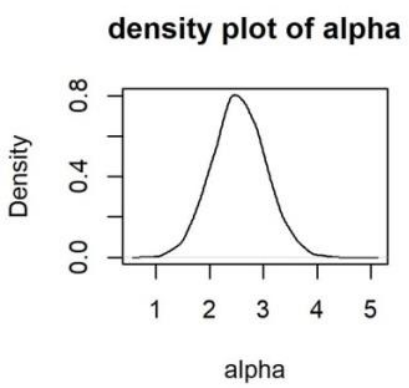

density plot of lambda

density plot of beta
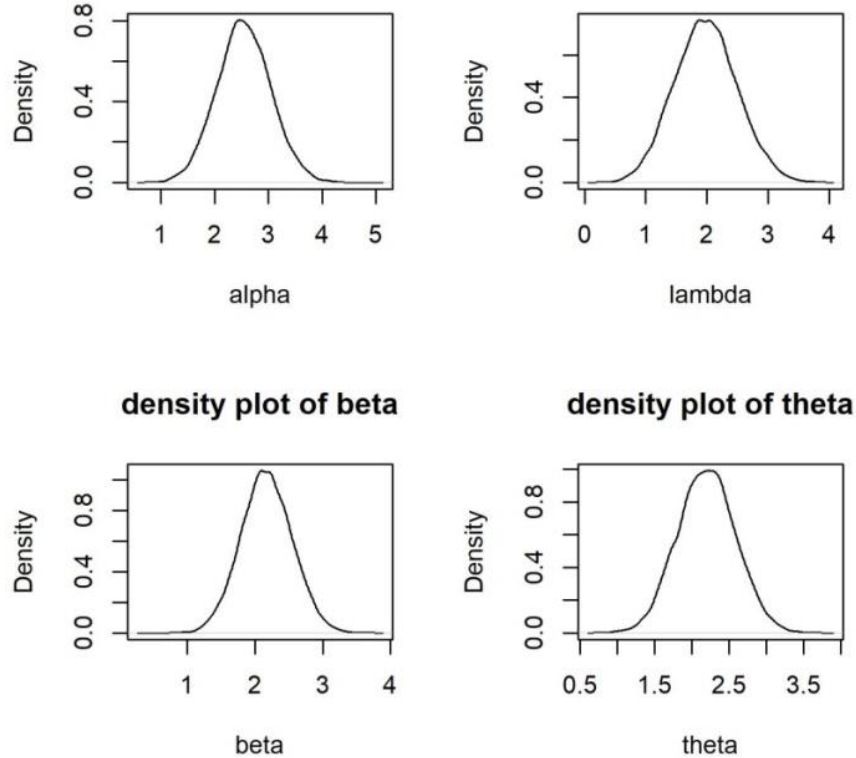

density plot of theta

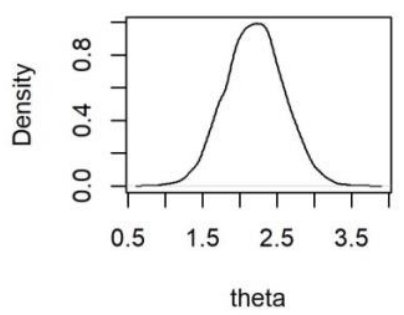

Figure 3. Density plots for $\alpha, \lambda, \beta, \theta$. 
International Journal of Mathematical, Engineering and Management Sciences

Vol. 4, No. 4, 882-894, 2019

https://dx.doi.org/10.33889/IJMEMS.2019.4.4-070

The Bayes estimates, Expected loss function (in brackets), 95\% HPD credible intervals (in brackets) and their length for stress strength reliability of Generalised inverted exponential distribution using informative and non-informative priors are shown in Table 3.

Table 3. Bayes estimates, expected loss functions (in brackets), HPD credible intervals (in brackets) with length for $\eta=P(Y<X)$ using non-informative prior under squared error loss function (SELF) and Generalised entropy loss function (GELF)

\begin{tabular}{|c|c|c|c|c|}
\hline & & GELF & GELF & HPD \\
& SELF & $b=0.5$ & $b=-0.5$ & credible interval \\
\hline Non-informative prior & 0.464897 & 0.443843 & 0.458252 & $(0.232979,0.651690)$ \\
& $(0.008732)$ & $(0.006339)$ & $(0.005043)$ & 0.418711 \\
\hline Gamma prior & 0.492506 & 0.484572 & 0.494069 & $(0.347330,0.636145)$ \\
& $(0.004417)$ & $(0.002621)$ & $(0.002551)$ & 0.288815 \\
\hline
\end{tabular}

The results obtained in case of simulation do hold in case of simulated data sets as well (Table 3). The 95\% HPD credible intervals in case of gamma prior have small width as compared to noninformative prior (Table 3). The value of expected loss function using gamma prior is smaller than using non-informative prior.

\section{Conclusion}

As sample size $n, m$ increases, the expected loss functions decreases as is seen from the Tables (1 and 2). The length of $95 \%$ HPD credible intervals decreases as sample size increases for the Bayes estimates of stress strength reliability of generalised inverted exponential distribution. As seen from the Tables (1 and 2), the length of credible intervals for gamma prior is less than for non-informative prior. The value of expected loss function using gamma prior is smaller than using non-informative prior.

It can be observed from the simulation study (Tables 1 and 2) that the expected loss function and the length of $95 \%$ HPD credible intervals in case of larger value of $\eta$ is smaller than taking small and medium values of $\eta$.

\section{Conflict of Interest}

The authors confirm that there is no conflict of interest for this publication.

\section{Acknowledgment}

The authors are thankful to the anonymous reviewers and the editor for their valuable suggestions and comments which has led to an improvement in the manuscript. We also like to acknowledge with thanks the financial assistance provided by the UGC, New Delhi, India. All the authors also acknowledge the support provided by DST under PURSE grant. 
International Journal of Mathematical, Engineering and Management Sciences

Vol. 4, No. 4, 882-894, 2019

https://dx.doi.org/10.33889/IJMEMS.2019.4.4-070

\section{References}

Abouammoh, A.M., \& Alshingiti, A.M. (2009). Reliability estimation of generalized inverted exponential distribution. Journal of Statistical Computation and Simulation, 79(11), 1301-1315.

Arnold, B.C., Balakrishnan, N., \& Nagaraja, H.N. (1998). Records. John Wiley and sons, New York.

Asgharzadeh, A., Valiollahi, R., \& Raqab, M.Z. (2011). Stress-strength reliability of Weibull distribution based on progressively censored samples. Statistics and Operations Research Transactions, 35(2), 103-124.

Baklizi, A. (2008). Likelihood and Bayesian estimation of $\operatorname{Pr}(\mathrm{X}<\mathrm{Y})$ using lower record values from the generalized exponential distribution. Computational Statistics \& Data Analysis, 52 (7), 3468-3473.

Calabria, R., \& Pulcini, G. (1996). Point estimation under asymmetric loss function for left truncated exponential samples. Communications in Statistics-Theory and Methods, 25(3), 585-600.

Chandler, K.N. (1952). The distribution and frequency of record values. Journal of the Royal Statistical Society: Series B (Methodological), 14(2), 220-228.

Chen, M.H., \& Shao, Q.M. (1999). Monte Carlo estimation of Bayesian credible and HPD intervals. Journal of Computational and Graphical Statistics, 8(1), 69-92.

Chopra, G., \& Ram, M. (2019). Reliability measures of two dissimilar units parallel system using gumbelhougaard family copula. International Journal of Mathematical, Engineering and Management Sciences, 4(1), 116-130.

Deepika, Singh, O., Anand, A., \& Singh, J.N.P. (2017). Testing domain dependent software reliability growth models. International Journal of Mathematical, Engineering and Management Sciences, 2(3), 140-149.

Dey, S. (2007). Inverted exponential distribution as a life distribution model from a Bayesian viewpoint. Data Science Journal, 6, 107-113.

Dey, S., \& Dey, T. (2014). On progressively censored generalized inverted exponential distribution. Journal of Applied Statistics, 41(12), 2557-2576.

Dey, S., \& Pradhan, B. (2014). Generalized inverted exponential distribution under hybrid censoring. Statistical Methodology, 18, 101-114.

Dey, S., Dey, T., \& Luckett, D.J. (2016). Statistical inference for the generalized inverted exponential distribution based on upper record values. Mathematics and Computers in Simulation, 120, 64-78.

Dube, M., Krishna, H., \& Garg, R. (2016). Generalized inverted exponential distribution under progressive first-failure censoring. Journal of Statistical Computation and Simulation, 86(6), 1095-1114.

Hassan, A.S., Muhammed, H.Z., \& Saad, M.S. (2015). Estimation of stress- strength reliability for exponentiated inverted Weibull distribution based on lower record values. British Journal of Mathematics \& Computer Science, 11(2), 1-14.

Hastings, W.K. (1970). Monte Carlo sampling methods using Markov chains and their applications. Biometrika, 57 (1), 97-109.

Hussian, M.A. (2013). On estimation of stress strength model for generalized inverted exponential distribution. Journal of Reliability and Statistical Studies, 6(2), 55-63.

Keller, A.Z., Kamath, A.R.R., \& Perera, U.D. (1982). Reliability analysis of CNC machine tools. Reliability Engineering, 3(6), 449-473.

Krishna, H., Dube, M., \& Garg, R. (2017). Estimation of P (Y < X) for progressively first failure-censored generalized inverted exponential distribution. Journal of Statistical Computation and Simulation, 87(11), 2274-2289. 
International Journal of Mathematical, Engineering and Management Sciences

Vol. 4, No. 4, 882-894, 2019

https://dx.doi.org/10.33889/IJMEMS.2019.4.4-070

Kumar, A., \& Ram, M. (2018). System reliability analysis based on Weibull distribution and hesitant fuzzy set. International Journal of Mathematical, Engineering and Management Sciences, 3(4), 513-521.

Li, J. (2016). Reliability calculation for dormant k-out-of-n systems with periodic maintenance. International Journal of Mathematical, Engineering and Management Sciences, 1(2), 68-76.

Li, J., Collins, G., \& Govindarajulu, R. (2019). System reliability growth analysis during warranty. International Journal of Mathematical, Engineering and Management Sciences, 4(1), 85-94.

Lin, C.T., Duran, B.S., \& Lewis, T.O. (1989). Inverted gamma as a life distribution. Microelectronics Reliability, 29(4), 619-626.

Mahmoud, M.A.W., El-Sagheer, R.M., Soliman, A.A., \& Abd Ellah, A.H. (2016). Bayesian estimation of P $[\mathrm{Y}<\mathrm{X}]$ based on record values from the lomax distribution and MCMC technique. Journal of Modern Applied Statistical Methods, 15(1), 488-510.

Metropolis, N., Rosenbluth, A.W., Rosenbluth, M.N., \& Teller, A.H. (1953). Equation of state calculations by fast computing machines. The Journal of Chemical Physics, 21, 1087-1092.

Nadar, M. \& Kizilaslan, F. (2014). Classical and Bayesian estimation of $P(X<Y)$ using upper record values from Kumaraswamy's distribution. Statistical. Papers, 55(3), 751-783.

Nadarajah, S. \& Kotz, S. (2000). Extreme value distributions: theory and applications. Imperial College Press, London.

Nelson, W. (1972). Graphical analysis of accelerated life test data with the inverse power law model. IEEE Transactions on Reliability, 21(1), 2-11.

Tarvirdizade, B., \& Garehchobogh, H.K. (2014). Interval estimation of stress-strength reliability based on lower record values from inverse Rayleigh distribution. Journal of Quality and Reliability Engineering, Volume 2014, Article ID 192072. 